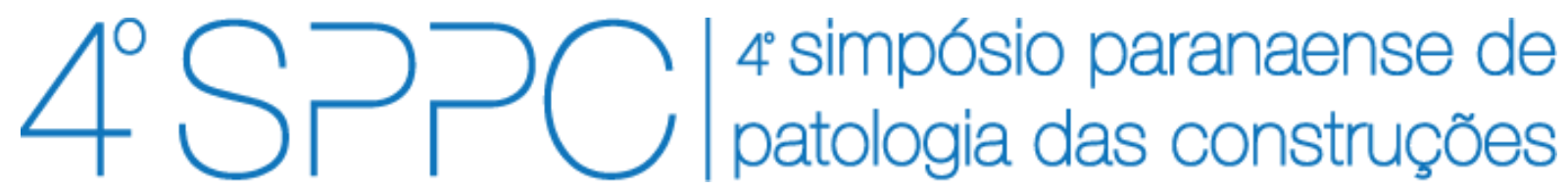

ISSN 2526-7248 artigo n. 4SPPC160, pp. 547-555, 2019

\title{
Uma revisão da literatura sobre ensaios acelerados para avaliação da formação de eflorescencias em argamassas
}

\author{
De Bem, Diogo Henrique ${ }^{1}$; Medeiros-Junior, Ronaldo Alves²; \\ ${ }^{1}$ Eng. Civil Mestre, Programa de Pós-Graduação em Engenharia de Construção Civil, \\ Universidade Federal do Paraná, diogobem@yahoo.com.br \\ ${ }^{2}$ Prof. Doutor, Universidade Federal do Paraná, medeirosjunior.ufpr@gmail.com
}

\begin{abstract}
Resumo: O sistema de revestimento está sujeito a diversas manifestações patológicas. Uma das manifestações comumente encontrada se trata da eflorescência, a qual se resume a depósitos salinos, normalmente de coloração branca, na superfície do revestimento, o que gera grande prejuízo, principalmente estético. Como a formação da eflorescência em condições normal é morosa, podendo demorar meses, ou mesmo anos, para ocorrer, este fato gera dificuldades para o estudo de maneiras de evitar o aparecimento desta manifestação patológica, bem como o impacto da utilização de diferentes materiais em sua formação. Apesar de existir uma norma americana de ensaio acelerado para formação de eflorescência em blocos cerâmicos, inexistem no momento normas nacionais ou internacionais com alguma metodologia de ensaio acelerado para argamassas. Este artigo teve como objetivo realizar uma revisão bibliográfica das diferentes metodologias de ensaio acelerado já praticada por outros autores. Com este trabalho foi possível observar que não há um consenso entre autores sobre a melhor metodologia de ensaio acelerado, visto a grande diversidade de métodos praticados nestes diferentes trabalhos pesquisados. Além disso, notou-se ainda que em grande maioria das publicações, a quantificação das eflorescências formadas era realizada somente por uma análise visual, o que torna o resultado empírico. Por esta razão, também se realizou uma revisão bibliográfica de diferentes métodos utilizados para quantificar as eflorescências e, pode-se observar que a quantificação através de softwares de análise gráfica se mostra uma opção viável.
\end{abstract}

Palavras-chave: Eflorescência, Ensaio Acelerado, Patologia, Argamassa

Abstract: The coating system is subject to various pathological manifestations. One of the most commonly encountered manifestations is efflorescence, which is basically saline deposits, usually white, that appear on the surface of the coating, generating great aesthetic damage. As the formation of efflorescence under normal conditions is slow, and may take months or even years to occur, this fact generates difficulties for the study of ways to avoid the appearance of this pathological manifestation, as well as the impact of the use of different materials in its formation. Although there is a US accelerated test for formation of efflorescence in ceramic blocks, there are currently no national or international standards with accelerated test methodology for mortars. This article aimed to perform a bibliographic review of the different methodologies of accelerated test already practiced by other authors. With this paper it was possible to observe that there is no consensus among authors about the best accelerated test methodology, given the great diversity of methods practiced in these studies. In addition, it was noted that in most publications, the quantification of the formed efflorescence was performed only by a visual analysis, which makes the result empirical. For this reason, a bibliographical review of different methods used to quantify efflorescence has also been carried out. It can be observed that the quantification through graphic analysis software is a viable option.

Keywords: Eflorescence, Accelerated Test, Building Pathology, Mortar 


\section{Introdução}

No Brasil, o uso de argamassas como forma principal de revestimento ainda é muito comum. Este fato, aliado à falta de manutenção adequada das fachadas e às chuvas constantes na região de Curitiba/PR, gera uma grande incidência de eflorescências nesta cidade. Marcondes e Moreira [1], por exemplo, perceberam que mais de $75 \%$ das fachadas de prédios com altura superior a 5 pavimentos na região do Batel em Curitiba apresentavam eflorescências.

A florescência se trata de um grupo de manifestações patológicas decorrente da formação de depósitos salinos. Sendo este deposito no interior da peça, caracterizase a formação de criptoeflorescências, já sendo na superfície destas, chamando-se neste caso de eflorescências [2]. Estes sais são principalmente de metais alcalinos (sódio e potássio) ou alcalino-ferrosos (cálcio e magnésio), podendo ser originados tanto dos materiais utilizados (cimento e tijolo), como do meio (solo, névoa salina, entre outros). O carreamento destes materiais para superfície ocorre por meio da água, proveniente de uma infiltração ou falta de estanqueidade adequada [3].

A portlandita é a principal fonte de cálcio nas eflorescências observados em obras em geral. Isso ocorre, pois, a lixiviação da portlandita ocorre com maior facilidade do que a dissolução de cristais de $\mathrm{C}-\mathrm{S}-\mathrm{H}$, além de estar presente na argamassa em maior quantidade do que os sais alcalinos [4]. A lixiviação deste composto da matriz cimentícia tende a gerar um aumento da permeabilidade e porosidade da argamassa, além também de reduzir suas resistências em até 20\%. [4-7].

Frente ao exposto, percebe-se que a eflorescência se trata de um problema crônico. Com foco em reduzir a incidência desta manifestação patológica, alguns autores [89] realizaram experimentos com adições de pozolanas, visto que estas possuem a propriedade de consumir hidróxido de cálcio durante a sua reação [10-13]. Contudo, a realização de ensaios com foco em avaliar a formação de eflorescência é complexa, visto a morosidade da formação de eflorescência em sua condição não acelerada e da possibilidade que nesta condição se forme criptoflorescências, ou seja, de a florescência se formar no interior da matriz cimentícia e não na superfície. A existência de ensaios acelerados se torna imprescindíveis para que seja possível a análise da interferência de diversas variáveis na formação desta manifestação patológica. Aberle et al. [14] comentam que, apesar de haver normatização para ensaio de eflorescência em blocos cerâmicos, ainda não existe um consenso sobre qual a melhor metodologia de ensaio para se avaliar a ocorrência de eflorescência em estruturas cimentícias, e nem como efetivamente quantificar os resultados destes ensaios.

Este artigo tem o objetivo de realizar uma revisão da literatura, apresentando algumas das metodologias de ensaios acelerados para formação de eflorescência e formas de posteriormente quantificar esta formação.

\section{Metodologia}

O procedimento de revisão bibliográfica utilizado neste estudo consistiu na compilação de duas pesquisas distintas. A primeira consistiu de todos os artigos encontrados por estes autores que utilizaram ensaios acelerados para formação de 
eflorescência em materiais cimentícios. Posteriormente, pesquisou-se publicações que utilizaram diferentes metodologias para quantificar a formação de eflorescência, sendo esta formação não necessariamente devido a utilização de algum ensaio acelerado. Devido a escacez de trabalhos sobre o assunto, considerou-se para esta análise um periodo de 1950 até 2018. A busca pelos artigos foi realizada na plataforma do Scholar Google, o qual realiza a pesquisa em diversos portais, como Elsevier, Springer, dentre outros.

\section{Revisão bibliográfica}

\subsection{Ensaios Acelerados}

A norma americana ASTM C67-92a [15] propõe uma metodologia de ensaio acelerado para avaliação da formação de eflorescências em blocos cerâmicos. Esta metodologia é composta basicamente por imergir $2,54 \mathrm{~cm}$ (1 polegada) do bloco cerâmico em água destilada durante 7 dias em ambiente com temperatura de $24 \pm$ $8^{\circ} \mathrm{C}$ e umidade relativa entre 30 e $70 \%$. Após este prazo, este bloco deve ser seco em estufa a $100^{\circ} \mathrm{C}$ por $24 \mathrm{~h}$ e depois visualmente comparado com outro bloco cerâmico deixado no mesmo ambiente, mas sem contato com água.

Conforme comentado anteriormente, inexiste uma norma para ensaio acelerado de eflorescência em argamassas. Diversos autores já sugeriram na literatura variados métodos para acelerar a formação desta manifestação patológica. O primeiro trabalho encontrado pelos autores deste artigo data do ano de 1955. Com foco em observar o impacto de diferentes teores de cimento e cal na incidência de eflorescência, Ritchie [16] moldou corpos de prova (CPs) cilíndricos de 50x100mm e os submeteu a 10 ciclos de molhagem e secagem, compostos de $14 \mathrm{~h}$ de submersão completa em água e 7 horas de secagem. $O$ autor conseguiu satisfatoriamente formar eflorescência nestas argamassas neste prazo.

Hennetier et al. [17], por sua vez, estudaram a formação de eflorescência em um conjunto misto composto por um bloco cerâmico revestido com $5 \mathrm{~mm}$ de argamassa nas suas faces laterais e inferior. Os autores submeteram estes conjuntos a quatro diferentes condições de ensaio, conforme distrito a seguir, e notaram que houve incremento da quantidade de eflorescência formada tanto com o aumento da temperatura, como com a utilização de pressão. Entretanto, a pressão influenciou mais do que a temperatura na geração desta manifestação patológica.

a) Condição 1: blocos mantidos sobre pressão de $200 \mathrm{~mm}$ de coluna de água, em ambiente de $23^{\circ} \mathrm{C}$ e com umidade relativa entre 50 e $70 \%$.

b) Condição 2: blocos mantidos em $2,54 \mathrm{~cm}$ de água em temperatura de $33^{\circ} \mathrm{C}$ e umidade relativa entre 30 a $50 \%$.

c) Condição 3: Blocos mantidos sobre pressão de $200 \mathrm{~mm}$ de coluna d'água, em ambiente com temperatura de $23^{\circ} \mathrm{C}$ e umidade relativa entre 30 e $50 \%$.

d) Condição 4: Blocos mantidos sobre pressão de $200 \mathrm{~mm}$ de coluna d'água, em ambiente com temperatura de $33^{\circ} \mathrm{C}$ e umidade relativa entre 30 e $50 \%$. 
Quarcioni et al. [18] estudaram outras 4 diferentes condições de ensaio com foco em acelerar a formação de eflorescência em CPs prismáticos (40x40x40mm) de argamassas mistas (cimento, cal e areia):

a) Condição 1: argamassa parcialmente imersa em água e o conjunto exposto a um ambiente controlado com umidade relativa de $60 \pm 5 \%$ e temperatura de $23 \pm 2^{\circ} \mathrm{C}$ por 7 dias.

b) Condição 2: argamassa encapsulada lateralmente em um recipiente plástico, com corpo de prova imerso em água até metade da sua altura e o conjunto exposto a temperatura e umidade do verão paulista por 7 dias.

c) Condição 3: argamassa parcialmente encapsulada, com corpo de prova imerso em água até metade de sua altura e conjunto mantido em estufa a $65^{\circ} \mathrm{C}$ durante 24 horas, visando simular a exposição de fachadas à temperatura elevada do verão.

d) Condição 4: argamassa parcialmente imersa em água, com face superior exposta a radiação térmica, em regime cíclico. Os corpos de prova foram mantidos em uma lâmina de cerca de $5 \mathrm{~mm}$ de água deionizada. Os CPs recebiam diariamente 10 horas de radiação térmica por meio de lâmpadas durante 7 dias (temperatura aproximada de 60 a $85^{\circ} \mathrm{C}$ ).

Quarcioni et al. [18] notaram, após 7 dias de exposição ao ensaio acelerado, que não houve aparecimento de eflorescência a base de cálcio (determinado por ensaio de DRX) sob as condições 1, 2 e 3 . Somente a condição 4 conseguiu satisfatoriamente formar eflorescência de carbonato de cálcio.

Uma metodologia bem distinta das demais foi utilizada Aberle et al. [14]. Estes autores aplicaram $2 \mathrm{~mm}$ de argamassa sobre uma telha absorvente e esta foi posteriormente colocada sobre um aparelho criado especialmente para este teste. Este aparelho foi mantido dentro de uma câmara com temperatura constante de $7^{\circ} \mathrm{C}$ e umidade relativa de $80 \%$, por 7 dias. Pelo interior deste aparelho circulava água a $20^{\circ} \mathrm{C}$. Segundo os autores, esta diferença entre o ambiente interno e externo da caixa criou dois microclimas diferentes em termos de temperatura e umidade, causando condensação, fluxo capilar e evaporação.

Kani et al. [19], por sua vez, analisaram a formação de eflorescência através da

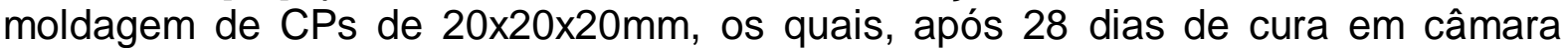
úmida, foram submersos em $40 \mathrm{ml}$ de água e mantidos nesta condição até a completa evaporação desta água (cerca de 2 semanas). Após este prazo, aguardou-se mais 2 semanas para os CPs secarem por completo e analisou-se visualmente a formação de eflorescência.

Visando avaliar o impacto do tempo de cura e do tempo de exposição ao ensaio acelerado na formação de eflorescência, Yao et al. [20] moldaram CPs de $20 \times 20 \times 20 \mathrm{~mm}$ e os deixaram em cura por diferentes períodos de tempo $(7,28$ e 60 dias). Após este prazo, submergiram os CPs em 10mm de água durante 7, 28 e 60 dias de exposição. Estes autores notaram que quanto maior o período de cura, menor a quantidade de eflorescência formada. Por outro lado, quanto maior o tempo 
DE BEM, D.H.; MEDEIROS-JUNIOR, R.A. UMA REVISÃO DA LITERATURA SOBRE ENSAIOS ACELERADOS PARA AVALIAÇÃO DA FORMAÇÃO DE EFLORESCENCIAS EM ARGAMASSAS. $4^{\circ}$ Simpósio Paranaense de Patologia das Construções (40 SPPC), artigo 4SPPC160, pp. 547 - 555, 2019. DOI: 10.4322/2526-7248.076

de exposição ao ensaio acelerado, maior a quantidade desta manifestação patológica.

Pernicová [21] e Kang e Kwon [22] também utilizaram uma metodologia de submersão parcial. Pernicová [21] imergiu $5 \mathrm{~mm}$ de CPs de 7 dias de idade de 150x150x150mm em água destilada e os manteve por 7 dias dentro de uma câmara a $24 \pm 1^{\circ} \mathrm{C}$, variando a umidade relativa entre 30 e $70 \%$. A diferença de umidade iria supostamente gerar períodos de evaporação e de lixiviação (ascensão de água pelos poros). Já Kang e Kwon [22] utilizaram CPs de 50x50x50mm, com 28 dias de cura, os quais foram mantidos por 14 dias em ambiente com temperatura de $7^{\circ} \mathrm{C}$ e $50 \%$ de umidade.

Outros autores, como Wei e Sutan [23] e Xue e Qian [23], inverteram a posição da lamina de água e, ao invés de colocar os CPs parcialmente submersos, estes colocaram a lâmina sobre os CPs. Wei e Sutan [23] utilizaram uma lâmina de $10 \mathrm{~mm}$ de água sobre a superfície dos CPs, 24 horas após moldados. Havendo a evaporação desta água, esta era recolocada até os 90 dias. Estes autores fizeram raspagem das eflorescências formadas em diferentes idades, mas não fizeram uma quantificação de áreas afetadas. Já Xue e Qian [24] utilizaram uma lâmina de 2 a $3 \mathrm{~mm}$ e somente repuseram a lâmina 3 vezes.

Weng et al. [8] moldaram CPs de 150x150x30mm e, devido à incerteza da melhor maneira de acelerar a formação de eflorescência, submeteram estes CPs a outras três diferentes condições de ensaio e perceberam que houve maior aparecimento de eflorescências em temperaturas baixas (condição c).

a) Condição 1: ambiente com 25ำ e $85 \%$ de umidade;

b) Condição 2: ambiente com $100 \%$ de saturação de dióxido de carbono, $100^{\circ} \mathrm{C}$ e $90 \%$ de umidade;

c) Condição 3: ambiente com temperatura entre $-5^{\circ} \mathrm{C}$ e $0^{\circ} \mathrm{C}$ e $20 \%$ de umidade.

Dessa forma, embora todas envolvam algum tipo de exposição a água, observa-se que as metodologias apresentadas na literatura são variadas para tentar acelerar a formação de eflorescência em materiais cimentícios.

\subsection{Quantificação da Eflorescência}

$\mathrm{Na}$ literatura, parte considerável das publicações envolvendo a formação de eflorescência realizaram uma quantificação somente visual. Este fato torna os resultados subjetivos e dependentes da capacidade de observação do realizador do ensaio.

Visando reduzir esta empiricidade, alguns autores utilizaram recursos tecnológicos para auxiliar na quantificação das eflorescências formadas. Hennetier et al. [17], por exemplo, utilizaram um analisador de imagens chamado Quantimet 500, o qual fez a quantificação por meio de análise de tons de cinza. 

4SPPC160, pp. 547 - 555, 2019. DOI: 10.4322/2526-7248.076

Por outro lado, Jang et al. [25] utilizou um espectrofotômetro (Minolta CM-2600) para realizar esta quantificação através de variações nos parâmetros de luminosidade.

Outros autores, como Farreira e Bergmann [26], Vázquez et al. [27] , Weng et al. [8] e Kang e Kwon [22], quantificaram a formação de eflorescência utilizando softwares de análise de imagem, como Image Tools 3.0, Geojars-10, METLAB e Paint.net, respectivamente.

Dessa forma, os estudos mais atuais tendem a utilizar o conceito de diferenciação de tonalidades a partir de algum software de tratamento de imagens para isolar as áreas com eflorescência e calcular a sua representatividade sobre a face avaliada.

\section{Conclusões}

Conforme pode-se observar, não há consenso entre os autores sobre a melhor metodologia para aceleração do processo de formação de eflorescência. Entretanto, pode-se realizar as seguintes observações:

- Aparentemente a utilização de uma pressão de coluna de água, conforme realizado por Hennetier et al. [17], gerou um aumento da quantidade de eflorescência formada, possivelmente devido a um incremento da lixiviação dos sais e por evitar que estes se depositem dentro da peça analisada, formando criptoeflorescências;

- A utilização de ciclos de temperatura, da mesma forma que realizado por Quarcioni et al. [18] na condição 4, tende a propiciar períodos em que há uma maior ascensão de água e lixiviação (período sem calor), seguido por períodos de evaporação (períodos com calor). O aumento da eflorescência nestes períodos de calor, possivelmente devido a uma maior evaporação, também foi notado por Hennetier et al. [17]. Entretanto, é importante ressaltar neste caso que o uso de temperaturas altas tende a aumentar a taxa de evaporação, o que pode aumentar também a formação de criptoeflorescências, dificultando a quantificação posterior.

- A utilização de temperaturas baixas tende a aumentar a formação de eflorescência, conforme observado por Weng et al. [8]. Isso possivelmente ocorre devido ao fato de que o hidróxido de cálcio apresenta uma maior solubilidade em baixas temperaturas, conforme Helene e Pereira [28]. Entretanto, é importante observar que também há necessidade de uma condição de evaporação, para que possa haver deposito de sais. Este fator foi criado por Weng et al. [8] ao se reduzir a umidade para $20 \%$.

- Nota-se, entretanto, que alguns autores conseguiram satisfatoriamente formar eflorescências com a simples submersão parcial em água destilada [19-22] ou atreves de ciclos de molhagem [22, 24], enquanto o mesmo não ocorreu nos ensaios realizados por Quarcioni et al. [18]. Possivelmente isso tenha ocorrido por diferença nas composições e características das argamassas estudadas. 
- Com relação à quantificação das eflorescências, observa-se que utilização de softwares de análise gráfica são uma alternativa interessante e relativamente simples para tornar esta tarefa menos empírica. A utilização de sistemas mais complexos, como com o uso do espectrofotômetro, também é possível, entretanto, a um custo superior.

Compilando todas as observações descritas nesta conclusão, pode-se realizar a sugestão de um método de aceleração da eflorescência para trabalhos futuros. Recomenda-se testar o uso concomitante de pressão com um ciclo de temperatura. O uso de pressão aumentaria a taxa de percolação de água e lixiviação. Já o ciclo de temperatura, variando de valores próximos a $0^{\circ} \mathrm{C}$ e $40^{\circ} \mathrm{C}$, auxiliariam a aumentar a solubilidade e lixiviação do hidróxido de cálcio e a taxa de evaporação, respectivamente. Sugere-se que a quantificação seja feita por meio de um software de análise gráfica, como o MATLAB.

\section{Agradecimentos}

Os autores agradecem a CAPES, ao CNPq, a Fundação Araucária e ao Programa de Pós-Graduação em Engenharia de Construção Civil (PPGECC) da UFPR.

\section{Referências}

[1] Marcondes, C.G.N.; Moreira, C.A.S. (2016) Levantamento quantitativo de manifestações patológicas de descolamento e eflorescência em fachada de edifícios com revestimento cerâmico no bairro batel e imediações de Curitiba. Revista Técnico-Científica CREA-PR, vol. 4, pp. 1-14.

[2] Kresse, P.(1987_Efflorescence-mechanism of occurrence and possibilities of prevention. Beton und Fertigteil Technik, vol. 53, pp.160-168. Alemanha, 1987.

[3] Bauer, L. A. F.(2009) Materiais de Construção: novos materiais para construção civil. $4^{\underline{a}}$ ed. v1. LTC.

[4] Choi, Y.S.; Yang, E.I.(2013) Effect of calcium leaching on the pore structure, strength, and chloride penetration resistance in concrete specimens, Nuclear Engineering and Design, vol. 259, pp. 126-136.

[5] Carde, C; François, R. (1999) Modelling the loss of strenght and porosity increase due to the leaching of cement pastes. Cement \& Concrete Composites, vol. 21, pp. 181-188.

[6] Ulm F.J.; Heukamp, F.H.; Germaine, J.T. (2001) Durability Mechanics of Calcium Leaching of Concrete and Beyond, Fracture Mechanics of Concrete Structures, Balkema Publishers, pp. 133-143, Lisse.

[7] Agostini, F.; Lafhal, Z.; Skoczylas, F.; Loodsaveldt, H.; (2007) Experimental study of accelerated leaching on hollow cylinders of mortar, Cement and Concrete Research, n.37, pp 71-78. 
[8] Weng, T.; Lin, W.; Cheng, A. (2013) Effect of Metakaolin on Strenght and Efflorescence Quantity of Cement-Based Composites. The Scientific World Journal, vol. 2013, pp. 1-11.

[9] Delair, S.; Guyonnet, R.; Govin, A.; Guilhot, B. (2007), Study of efflorescence forming process on cementitious materials. 5th International conference on concrete under severe conditions of environmental loading, França.

[10] Khater,H., (2010) Influence of Metakaolin on resistivity of cement mortar to manesium chloride solution, Ceramics, vol 54, pp. 325-333.

[11] Saito, H.; Degushi, A. (2000) Leaching tests on different mortars using accelerated eletromechenical method. Cement and Concrete Research, vol. 30, pp. 1815-1825.

[12] Trník, A.; Scheinherrova, L.; Cerny, R.(2017) Influence of Silica fume on the hydration of Cement Pastes Studied by Simultaneous TG-DSC Analysis, International Journal of Civil and Environmental Engineering, vol. 11 (8).

[13] Medeiros, M.; Raisdorfer, J.W.; Hoppe, J.; Medeiros-Junior, R.A. (2017) Parcial replacement and addition of fly ash in Portland Cement: incluence on cabonation and alkali reserve, Journal of Building Pathology and Rehabilitation.

[14] Aberle, T.; Keller, A.; Zurbriggen, R. (2007) Efflorescence. Mechanisms of formation and ways to prevent. $2^{\circ}$ Congresso Nacional de Argamassas para Construção - APFAC, Lisboa.

[15] ASTM International. ASTM C67 - 92A: (1992) Standard test methods of sampling and testing brick and structural clay tile, Philadelphia.

[16] Ritchie, T. (1955) Study of Efflorescence Produced on Ceramic Wicks by Masonry Mortar, Journal of the American Ceramic Society, vol. 38 (10), pp. 362-366.

[17] Hennetier, J.; Alameira, J.V.; Correia, A.M.S; Ferreira, V.M. (2001) Efflorescence and its quantification in ceramic building materials. British Ceramic Transactions, vol 100 n.2. Londres.

[18] Quarcioni, A.; Chotoli, F. E Aleixo D. (2003) Ensaio acelerado para simular a formação de eflorescência em argamassas endurecidas. V SBTA - São Paulo.

[19] Kani, E.; Allahverdi, A.; Provis, J. (2012) Efflorescence control in geopolymer binders based on natural pozzolan, Cement \& Concrete Composites vol. 34, pp. 25-33.

[20] Yao, X.; Yang, T.; Zhang, Z. (2016) Compressive Strength development and shrinkage of alkali-activated fly ash-slag blends associated with efflorescence. Materials and Structures vol. 49. pp. 2907-2918.

[21] Pernicová, R. (2016) The susceptibility of forming efflorescence on concrete depending on the mold-releasing agent. Advanced Materials, Mechanical and Structural Engineering - pp. 151 - 154. Coréia do Sul.

[22] Kang, S.; Kwon, S.(2017) Effect of red mud and Alkali-Activated Slag Cement on efflorescence in cement mortar, Construction and Building Materials vol. 133, pp. 459-467. 

4SPPC160, pp. 547 - 555, 2019. DOI: 10.4322/2526-7248.076

[23] Wei, O.; Sutan, N. (2013), The Influence of Finely Ground Mineral Admixture (FGMA) on Efflorescence, USIMAS Journal of Civil Engineering, n. 4, pp 50-55.

[24] Xue, B.; Qian, C.(2015) Mitigation of efflorescence of wallboard by means of bio-mineralization, Frontiers in Microbiology vol. 20.

[25] Jang, H.; Kang, H.; So, S. (2014) Colour expression characteristics and physical properties of colored mortar using ground granulated blast furnace slag and White Portland Cement, KSCE Journal of Civil Engineering, vol. 4, pp 1125-1132.

[26] Ferreira, C.; Bergmann, C. (2010) Transport of $\mathrm{Ca}^{\wedge}(+2)$ and $[\mathrm{SO}] \_4^{\wedge}(-2)$ lons in Porous Media of Clay Product and Its Association with the Efflorescence Phenomenon, Transport in Porous Media vol. 85, pp. 905-917.

[27] Vázquez, M.; Galán, E.; Gerrero, M.A.; Oortiz, P. (2011) Digital image processing of weathered stone caused by efflorescence: A tool for mapping and evaluation of stone decay, Construction and Building Materials, vol. 25, pp. 1603-1611.

[28] Helena, P.; Pereira, F.(2007) Rehabilitación y Mantenimiento de Estruturas de Concreto, Patrocinio: Sika, São Paulo. 